\title{
Intelligent spacing selection model under energy-saving constraints for the selection of communication nodes in the Internet of Things
}

\author{
Jing-Shu Sun ${ }^{1} \cdot$ Teng Zhu $^{2} \cdot$ Marcin Wozniak ${ }^{3}$ (I) \\ Accepted: 8 July 2021 / Published online: 11 September 2021 \\ (C) The Author(s) 2021
}

\begin{abstract}
Current IoT communication node spacing selection process show may potential areas for improvements such as high delay ratio, high total energy consumption ratio, confusion of the optimal communication information band, intelligent spacing node design under the constraints of the energy-saving selection of IoT communication. Based on energy-saving constraints, the link status between nodes is evaluated through link stability and link quality. In order to prevent the generation of serious noisy nodes and frequency hopping data, the interference nodes under the intrusion of the Internet of Things are identified by determining transition amplitude of the noise nodes in the transmission data sequence. Finally, according to the calculation results of the optimal communication node selection, the design of the intelligent spacing selection model for the communication nodes of the Internet of Things is realized. The simulation results show that the established model not only reduces energy consumption of nodes, shortens the average transmission delay of nodes, but also improves anti-interference effect of node spacing selection.
\end{abstract}

Keywords Internet of Things $\cdot$ Communication nodes $\cdot$ Energy-saving constraints $\cdot$ Spacing selection $\cdot$ Link state evaluation . Interference nodes

\section{Introduction}

With the popularity of Internet of Things applications, the indicators that need to be considered for network deployment optimization often include node coverage quality, number of network nodes, node perception range, network operating cycle, etc., and with the diversification of Internet of Things applications, in various applications in the field, the Internet of Things also plays an important role. Its main branch, wireless sensor network, is scattered and deployed at application demand locations through various sensors [1]. For example,

Jing-Shu Sun

jingshu_sun_gz@163.com

Marcin Wozniak

marcin.wozniak@polsl.pl

Teng Zhu

teng_zhu_gz@163.com

1 Department of Electronic Information Engineering, Guangzhou College of Technology and Business, Guangzhou 528138, China

2 Guangdong Earthquake Agency, Guangzhou 510070, China

3 Faculty of Applied Mathematics, Silesian University of Technology, 44100 Gliwice, Poland the deployment and optimization of sensing nodes for the manufacturing Internet of Things enables enterprises to complete the effective coverage of the entire manufacturing process with limited cost investment, and achieve complete data sampling of the entire manufacturing process [2]. For the monitoring of various industries, agriculture and even harsh environments, wireless sensor network products can break through the traditional monitoring methods. While satisfying the flexibility, reliability and safety, it can reduce the cost for various environmental monitoring applications, and at the same time process of traditional monitoring is reduced, and it facilitates the acquisition of random data research [3]. At the same time, the efficient deployment strategy of sensing nodes has self-organization, fault tolerance, and dynamic adaptive adjustment capabilities under conditions of industrial and other manufacturing complex physical environments, thereby ensuring stability and reliability of data collection. Therefore, a good node deployment and node scheduling strategy has an important impact on network performance and network survivability [4].

In the Internet of Things, communication method uses certain data nodes for effective transmission. The optimal data node in communication process represents the completeness of communication, but the Internet of Things was often invaded, resulting in serious noise nodes, serial frequency nodes 
and frequency hopping node. In the selection of nodes, current methods can not completely and effectively remove interference nodes, so it is impossible to calculate the optimal communication nodes in cluttered nodes, and the effective selection of node spacing cannot be realized. Therefore, it is necessary to study selection method of communication node spacing in the Internet of Things $[5,6]$.

Reference [7] presents a model that in order to enhance communication quality after the fiber laser network is invaded. A method for selecting the optimal communication node based on improved Voronoi diagram is proposed, which uses the weighted Voronoi diagram method to obtain the optimal communication node position. The basic modeling principle of Voronoi diagram is analyzed, and the positioning model based on weighted Voronoi diagram is formed. The RSSI values collected by the unknown nodes in the fiber laser network are arranged in descending order, and the corresponding values are given by the node weight assignment. The weighted Voronoi diagram of each reference node is calculated through the node multi hop information. The weighted Voronoi diagram positioning algorithm is used to calculate the weighted area of the reference node and intersection of the weighted Voronoi diagram. The centroid of the intersection is optimal communication node. The experimental results show that positioning efficiency and anti noise performance of this method are high, which can improve communication efficiency of fiber laser network, but the optimal communication information wave domain is unstable, which affects stability of selection of communication nodes in the Internet of Things. Reference [8] proposed a distance aware clustering strategy in cellular IoT based on energy acquisition. By analyzing the IoT with stable energy acquisition sources and considering cooperation between energy and communication, a distance aware clustering strategy is proposed to balance the imbalance between node acquisition and energy consumption in the Internet of Things. According to the cluster head selection mechanism, the nodes are divided into cluster head node and cluster member node. The cluster head communicates with the base station directly, and the members send the service to the cluster head of the cluster, and then the cluster head forwards it to the base station. The results show that under the constraint of total energy consumption, the proposed method can reduce communication interruption by at least $50 \%$, but the problem of service delay ratio is not effectively solved. In addition to the above methods, some researchers have proposed an ant colony optimization based service node selection algorithm for content centric networks. Content Centric Network $(\mathrm{CCN})$ is a novel network architecture for content distribution through name based addressing and routing. Ant colony optimization is a heuristic algorithm, which can find the optimal solution by sending probe ants. By modeling the $\mathrm{CCN}$ service node selection problem, the distributed service node selection is realized. The simulation results show that the algorithm can significantly improve the routing performance and reduce the cache on CCN nodes, but the total energy consumption of the algorithm is large, and the practical application is not strong.

Aiming at the problems existing in methods, under the constraints of energy conservation, an intelligent spacing selection model for IoT communication nodes is designed. Select intelligent distance of IoT communication nodes under energy-saving constraints to reduce the overall energy consumption of node selection. Consider relation between link maintenance time and transmission delay, evaluate the stability and quality of the links between nodes, and evaluate the Internet of Things. According to the results of the state evaluation of the links between nodes, the transfer characteristic model of the communication nodes is constructed. This model is used to confirm frequency hopping signal and time sequence of the intrusion of the Internet of Things, realize the marking of the interference node, and reduce the influence of confusion of information frequency band on the selection of node spacing. Finally, establish model assumptions and design the optimal intelligent distance selection model.

\section{Communication node selection method under energy conservation constraints}

Study the method of selecting IoT communication nodes under energy-saving constraints. First, fully consider the link status and link quality, and construct energy-saving constraints targeting low power consumption, high throughput and high reliability IoT communications, and link stability Performance evaluation and link quality evaluation mark the interference node under the intrusion of the Internet of Things, set the coordinate parameters of the communication node, determine the communication node according to the effectiveness of the node load capacity, perform energy balance processing on the known effective area node, and finally in each communication area Calculate the original set and use the data transmission characteristics to select the optimal communication node.

\subsection{Energy-saving constraints}

Based on communication characteristics of the Internet of things, in order to solve problems such as disappearance of communication nodes and imbalance of communication among nodes in the network, and to achieve the goal of building a model with low power consumption, high throughput and high reliability, energy-saving constraints are proposed. General constraints can increase the average life of intelligent nodes in Internet of Things communication system, but the effect of energy-saving technology is limited [9, 10]. Therefore, in order to ensure that energy consumption of intelligent node due to data forwarding and transmission is 
lower than a certain energy consumption threshold, the remaining nodes are used to ensure that intelligent node can maintain normal operation in its design life cycle, and energy-saving constraints are proposed.

When selecting the next hop forwarding node, the link quality and link stability between one hop nodes are considered, which will increase the number of hops and easily lead to the problem of local maximization. Therefore, when selecting the next hop forwarding node, the effective node density is taken into account to avoid the above-mentioned problem [11]. When selecting the next hop node, fully considering the link state and link quality can not only effectively improve the packet delivery rate, but also greatly reduce the packet transmission delay time.

\subsection{Link state evaluation between nodes}

Traditional methods are difficult to find a reliable distance from the source node to the destination node. In order to ensure reliability of the distance from the source node to the destination node, the reliability of the link between each hop node must be guaranteed. Link state index is used to evaluate the link state between nodes. Link state is evaluated by link stability and transmission quality.

\subsubsection{Link stability evaluation}

The change of topology is often caused by the change of speed and direction, which seriously affects stability of the link [12]. In this paper, the change of relative displacement is used to measure stability of the link between nodes. The distance between nodes is obtained by the following formula:

$d=J_{i-1}(I-h)$

where, $I$ is the sending node; $h$ is the neighbor node. According to formula (1), the relative displacement between nodes is obtained:

$l=J_{t} \sum_{j=1}^{n}\left(I_{j}-h_{j}\right)$

where, $J_{t}$ is the distance between the sending node and the neighbor node at the moment $t$. By evaluating relative displacement change value between nodes, the stable state of the link can be obtained. The smaller the change value, the more stable the link.

\subsubsection{Link quality assessment}

Since the data packet sent by the sending node is much larger than the target data packet, a model based on video transmission is given, and the error rate of the node receiving packet is used to evaluate the link quality between nodes [13]. The energy of the signal is:
$E_{n}=\frac{\varepsilon\left(\eta_{j}+\eta_{n}\right)}{l \times r}$

where, $\eta_{j}$ is the received energy; $\eta_{n}$ is the transmitted energy; $\varepsilon$ is the transmission signal wavelength; $r$ is the path attenuation factor. According to the acquired signal energy, we calculate the signal-to-noise ratio of the received signal:

$L_{S N R}=E_{n} \times \frac{p(x, y)}{p(x)-p(y)}$

where, $p(x)$ is all the energy received; $p(y)$ is the energy containing noise.

Due to the link topology changes caused by the node speed and direction, it is easy to break the link when sending a packet, so it is very important to calculate reliable maintenance time of one hop link [14]. When the sending node receives a hello packet from a neighbor node, the link maintenance time between the sending node and the node is calculated:

$T=\sum_{i, j=1}^{l} v_{i} v_{j} y_{i} y_{j}\left(x_{i} \cdot x_{j}\right)$

where, $v_{i}$ is the node sending speed; $v_{j}$ is the speed of the node sending data packets. Then the end-to-end delay when sending a data packet is:

$T_{\text {delay }}=W_{k} \cdot T$

where, $W_{k}$ is the data packet transmission delay. When $T_{\text {delay }}$ $>T$, the communication link is extremely fragile and the link is unreliable. Therefore, considering the relation between the link maintenance time and the transmission delay, the link state between nodes can be judged.

\subsection{Interfering nodes under the intrusion of the Internet of Things}

Under the intrusion of the Internet of Things, it will cause the tampering of the original Internet of Things communication structure. The tampered Internet of Things will generate new transmission frequencies in the transmission channel, resulting in serious noise nodes and frequency hopping data [15]. Therefore, based on results of the state evaluation of the link between nodes in section 2.2, the communication node transfer characteristic model is constructed, the frequency hopping signal and time sequence of the Internet of Things intrusion are confirmed, and the interfering node is marked. The distribution of interference nodes is shown in Figure 1.

The distribution state of the interference data is consistent with the distribution state of the interference node, and the frequency hopping data transfer feature can be used to mark the data of the frequency hopping node. For noisy nodes, the 


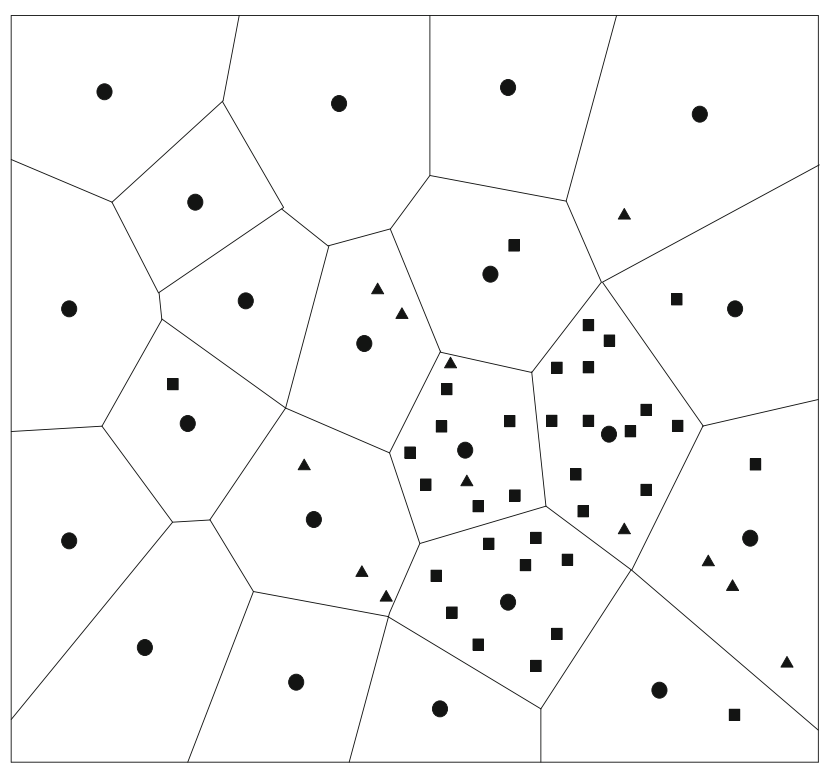

- Frequency hopping node $\mathbf{m}$ Noisy node $\Delta$ Other interference nodes

Fig 1. Distribution of interference nodes

model cannot be used to directly mark, therefore, it is necessary to use the distributed transmission data information feature to calculate the mark [16].

Assuming that the distribution structure of intrusion network nodes of the Internet of Things is a multivariate vector model, the intrusion information source is a group of diversified nodes. The distribution process is relatively random, and noise nodes will be randomly mixed into communication nodes. Therefore, the interference factor is used to calculate the noise nodes. Suppose the interference term is $G_{i}$, then the communication node transfer characteristic modeling receiving noise data signal node is:

$x(n)=\sum_{i=1}^{n} T\left(R k_{i}-G_{i}\right)$

where, $x(n)$ is the noise node; $R$ is the distributed coefficient of the Internet of Things; $k_{i}$ is the ability value of the Internet of Things to receive noise data.

Use the least squares estimation algorithm [17] to perform data spectrum cognitive detection on the calculated noise node, obtain the transition feature quantity of the noise node, and determine the transition amplitude of the noise node in the transmission data sequence as:

$\varphi(n)=\sum_{i=1}^{n} x(n)\left(y^{\prime}-R y^{2}\right)$

where, $\varphi(n)$ is the transition amplitude of the noise node; $y^{\prime}$ is the degree of spectral separation of the noise node; $y^{2}$ is the nonlinear time series coefficient.

Combining the transition characteristic equation with the transition amplitude of the noise node, the state of the noise node can be confirmed. The node meeting the above state is regarded as the marked noise node, and the interference node under the intrusion of the Internet of Things can be effectively identified through the model.

\subsection{Optimal communication node selection calculation}

The interference node marking under the intrusion of the Internet of Things has provided preconditions for the intelligent distance selection of Internet of Things communication nodes, but in order to further improve the selection effect, the optimal communication node needs to be calculated. Communication node selection process:

1. First, the algorithm must be initialized and optimized to ensure accuracy of the optimal communication node setting area, set the code of the network node interface, and set the coordinate parameter of the communication node.

2. Secondly, divide the communication area, choose transmission interface in the two-dimensional area of the Internet of Things, record the coordinate value of the Internet of Things communication, obtain the communication cluster head information, calculate the number of communication nodes, and then determine communication node based on the node load capacity effectiveness, the energy balance processing of the known effective regional nodes to ensure the correctness of the cluster head selection [18].

3. Finally, the original set is calculated in each communication area, and the optimal communication node is selected using data transfer characteristics.

The optimized communication node selection can improve the computing power of the calculation method. Set the number of types of communication nodes to $m$, and use $m_{j}$ to represent the type $j$ communication node. If there is a change in communication network architecture under the intrusion of the Internet of Things, a node classification data set $B$ is established, which includes $g$ node data, $B=\left\{b_{1}, b_{2}, \ldots\right.$, $\left.b_{g}\right\}$. Therefore, the goal of the optimal selection algorithm is to select the extreme value of $b_{g}$. Use formula (9) to filter ineffective communication nodes in the set:

$S_{\varphi_{n}}=H+\sqrt{H^{2}-x(n)}$

where, $H$ is the prior probability of the communication node.

In order to make the prior probability and the node to have the same stability under energy-saving constraints, the interference factors of time series arrangement are added. Lagging node is filtered out, and extreme value processing is performed to obtain the optimal communication node: 
$\partial_{\xi}=H\left(\xi^{T} D-\xi^{T} \xi\right)-H\left(\varphi_{n} \times B_{j}\right)$

where, $\partial_{\xi}$ is the optimal communication node; $B_{j}$ is the communication node selected at the position $j$. Applying formula (10) to calculate the optimal communication node of the Internet of Things we can intelligently obtain the optimal communication node distance, reduce the energy consumption of Internet of Things communication, and improve the efficiency of Internet of Things communication.

\section{Intelligent spacing selection model for loT communication nodes}

Based on the calculation results of optimal communication node selection in Section 2, the intelligent distance selection model for IoT communication nodes is constructed by calculating the distance between nodes.

\subsection{Calculation method of distance between nodes}

Because in the Internet of Things, different attributes of each node have different effects on the calculated distance between nodes. The greater the distance between two nodes, the lower similarity, and the lower probability of being in a small group $[19,20]$. Therefore, when calculating the feature distance between nodes, it should be multiplied by weight coefficient of the attribute. The larger the weight coefficient, the greater the influence of the attribute.

The distance from a node to a cluster represents the distance between a node and a super node. The smaller the distance, the higher the similarity between the node and the super node, and the more it should be divided into the cluster. The calculation method is:

$W_{\text {dist }}=\sqrt{w\left(k_{1}, k_{2}\right)^{2}+d\left(k_{1}, k_{2}\right)^{2}}$

Sort all points in the point set according to the aggregation density, and take the point with the highest aggregation density as the first initial center. At the same time, seed nodes that are far apart as more representative, and nodes in the same cluster can be avoided as seed nodes [21]. Therefore, in areas with high aggregation density, distant points are selected as seed nodes. The optimization algorithm of the initial node is as follows:

(1) Obtain high-density area of nodes and get the set $D_{\text {high }}$;

(2) Select the node with the highest aggregation density as the initial central node;

(3) Select the point with the highest density in set $D_{\text {high }}$ as the seed node.

Since the privacy protection strength of existing network nodes is $u$, the number of initial nodes can be expressed as $n / u$. However, the number of nodes in each cluster in this method is different. Therefore, the number of initial nodes is not a fixed value. The value range is:

$n / D_{\text {high }} \leq D_{\text {num }} \leq n / k$

According to the range of distance between nodes shown in formula (12), the distance between reference node and node in the nearest layer and the distance between reference node and nearest layer node are preliminarily calculated. According to this, the interfering nodes under the intrusion of the Internet of Things are identified and removed, and the real-time and accuracy of communication between the sensors in the Internet of Things are improved.

\subsection{Optimal intelligent distance selection model}

The model assumes that, when each sensor node in the Internet of Things cannot communicate in time, the data collected by all sensor nodes is saved in the cache sequence [22], and the node enters into sleep state. When each sensor node resumes normal communication, each sensor node enters working state, and the sensing data is sent to the Internet of Things Sink node by hop communication method.

On the basis of the above model assumptions, the monitoring area of the Internet of Things is divided into $\mathrm{n} \times \mathrm{n}$ grids of equal size. All grids are coded uniformly according to the principle of top-down and left to right. Sink nodes can move to any grid center to collect data. Assuming that $o$ represents the set of all grid centers after coding, and $o_{n}$ represents the status indicator of the Internet of things Sink node staying in the network center, the number of grid positions that sink nodes need to stay in is as follows:

$N_{o}=\sum_{i=1}^{n} P\left(V_{l}\right)-P\left(V_{s}\right)$

where, $P\left(V_{l}\right)$ is the data collected for a period of time when the sink node of the Internet of Things moves to the center of the grid; $P\left(V_{s}\right)$ is the data collected without staying.

Suppose that the sink node of the Internet of Things collects data along a certain paths from a certain grid center position, and the time required to finally return to the initial grid center position is a data collection cycle, represented by $T_{h}$. The specific calculation formula is as follows:

$T_{c}=\frac{A\left(V_{l}, V_{s}\right)}{\left(e^{2}-1\right)} T_{f}\left(V_{l}, V_{s}\right)$

where, $T_{f}$ is the dwell time of the sink node of the Internet of Things at the grid location; $e^{2}$ is the set of all the staying grid locations; $A$ is the movement rate of the sink node of the Internet of Things.

In order to ensure that the data collected by the sink node of the Internet of Things can cover all sensor nodes, set each 
sensor node to stay within the single-hop maximum communication range of a grid position, and the following conditions are met:

$d(i, j)>W\left(V_{\max }, V_{\min }\right)$

where, $d(i, j)$ is the distance between sensor node $i$ and node $j$ under the Internet of Things; $V_{\max }$ is the maximum communication distance between sensor nodes; $V_{\min }$ is the minimum communication distance between sensor nodes.

Under the above constraints, assume that $I_{\varepsilon}$ and $I_{\tau}$ respectively represent the energy consumed when the node sends 1-bit data and amplifies 1-bit data. Assuming that $\kappa_{i}$ represents the data sending rate of node $i$ when sink node stays at grid location $\kappa, p_{i \omega}$ represents the set of all staying grid locations where communication distance to node $i$ in the Internet of Things is less than the maximum single-hop communication range of the node, calculated in one during the data collection period, the data transmission energy consumption of node $i$ in the Internet of Things is:

$E_{i}=I_{\varepsilon}\left(t-t_{0}\right) I_{\tau}\left(t_{0}\right)+\kappa_{i}{ }^{\left({ }^{--\tau}\right)}+p_{i \omega}$

According to the above analysis, the intelligent spacing selection model of communication nodes under the Internet of Things is constructed. The specific modeling formula is as follows:

$$
\begin{aligned}
S_{R} & =p(x, y) \log _{r} \frac{p(x, y)}{p(x), p(y)} \times E_{i} \\
& =p(x, y)\left(\log _{r} p(x, y)-\log _{r} p(x)-\log _{r} p(y)\right) \times E_{i}
\end{aligned}
$$

As shown in formula (17), under the constraints of energy saving, the intelligent distance selection model of IoT communication nodes is judged by the link state between nodes through the link state evaluation method between nodes. According to the judgment result, the model is established to identify objects. Interfering nodes under network intrusion, avoid serious noise nodes and frequency hopping data. Finally, based on the calculation result of the optimal communication node selection, the intelligent distance selection model of the communication node of the Internet of Things is realized.

\section{Experimental study}

In order to verify comprehensiveness and effectiveness of the intelligent distance selection model under the energy-saving constraint of the communication node selection of the Internet of Things, the experimental verification is carried out. In order to verify the advantages of the proposed method, the optimal communication node selection method based on improved Voronoi diagram (Method 1), distance aware clustering strategy based on energy acquisition (Method 2) and content center network service node selection algorithm based on ant colony optimization (Method 3) are compared for performance comparison.

\subsection{Experimental environment and parameter settings}

In order to ensure the rigor of the experimental process, the different ways of intruding the Internet of Things during the experiment lead to different random collocation methods of interfering nodes. Therefore, the experimental process has a certain degree of randomness. In order to make the experiment more scientific, the experimental data parameters should be within the controllable range. Since the experimental process is a comparison of multiple methods, the controlled variable method is selected for the experiment. The specific simulation parameter settings are shown in Table 1.

\subsection{Experimental indicators}

Select the following three indicators for performance evaluation:

(1) Delay ratio

The delay ratio is the ratio between the time delay generated and the time delay not generated when the IoT communication node is selected. The delay ratio is an important indicator to measure the performance of the method. The calculation formula is as follows:

$\left(P_{M T}\right)=\frac{N}{k M}$

where, $M$ is the number of times that the node does not generate delay.

(2) Proportion of total energy consumption

The proportion of total energy consumption is the energy consumed to complete the selection of all nodes.

(3) Optimal communication information wave domain test

The optimal communication information wave domain experiment can verify the stability of the optimal communication node selection. There will be a very high bit error rate under the intrusion of the Internet of Things. Therefore, in the process of selecting the optimal communication node, the accuracy of node selection should be ensured. At the same time, the stability performance is investigated, the wave state can be observed through the waveform in the information wave domain, and its stability can be investigated according to the wave state of the wave spectrum. The information wave domains under different network structures are different. During the experiment, a variety of Internet of Things intrusion methods are selected, and the experimental results can be obtained by observing the state of the information wave domain. 
Table 1. Experimental parameter settings

\begin{tabular}{ll}
\hline Parameter & Value/Description \\
\hline Signal transmission speed & $3 \times 100 \mathrm{~m} / \mathrm{s}$ \\
Amplification factor & $10 \times 10-12 \mathrm{~J} / \mathrm{bit}$ \\
Number of nodes & 150 \\
Proportion of communication intelligent nodes & $80 \%$ \\
Packet size & $500 \mathrm{MB}$ \\
Energy consumption per bit & $50 \times 10-9 \mathrm{~J}$ \\
Network data link node connection processing mode & Randomly select nodes \\
Data processing delay & $2.5 \times 10-5 \mathrm{~S}$ \\
Disturbance parameter & $60 \mathrm{~J} / \mathrm{bit}$ \\
Interfering node invasion parameters & $60 \mathrm{~J} /(\mathrm{bit} \cdot \mathrm{s}-1)$ \\
Network communication port & 150 \\
Optimal channel load limit & $1.0 \mathrm{~J}$ \\
Network distribution vector & $20 \mathrm{pJ}(\mathrm{bit} \cdot \mathrm{m}-1)$ \\
\hline
\end{tabular}

\subsection{Analysis of experimental results}

The following is the comparison result of the delay ratio of different methods, as shown in Figure 2.

Analysis of Figure 2 shows that the highest value of the delay ratio of the proposed method when selecting nodes is less than $30 \%$, while the lowest value of the existing methods is higher than $30 \%$. In comparison, the advantages of the proposed method are obvious, it shows that the proposed method will greatly reduce the time delay when selecting communication node of the Internet of Things, and has a higher efficiency. This is because the method calculates the end-to-end delay when sending a data packet and judges the state of communication link, thereby avoiding the problem of increased delay ratio due to unreliable links.

The following is the comparison result of the total energy consumption ratio of different methods, as shown in Figure 3.

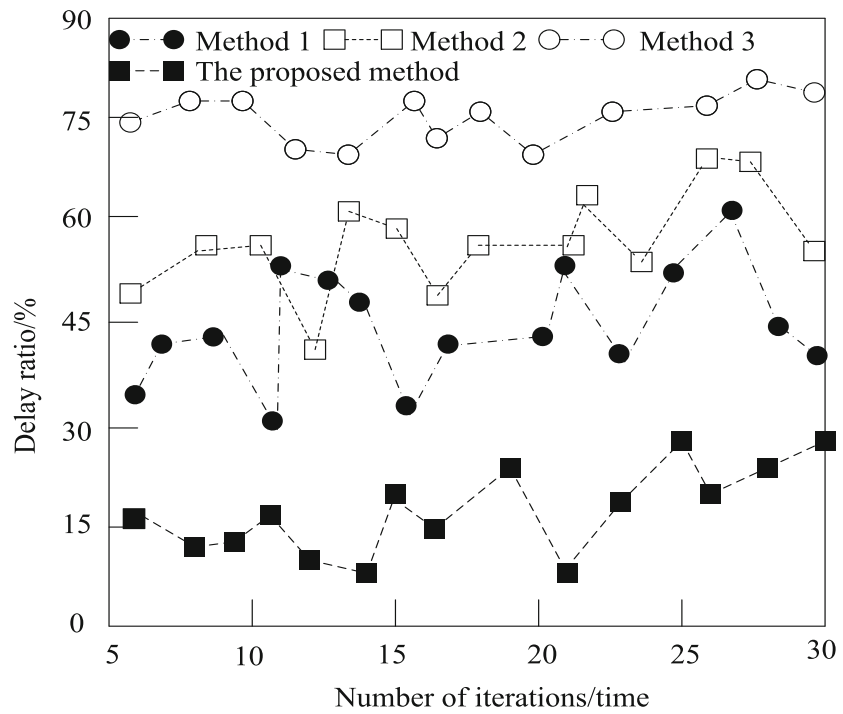

Fig 2. Comparison of delay ratios of different methods
Analysis of Figure 3 shows that the existing method performs poorly in terms of total energy consumption. Compared with the existing method, the proposed method has better performance in energy saving. This is because the proposed method is to select nodes under energy-saving constraints. The energy-saving constraints can ensure that the energy consumption of smart nodes due to data forwarding and transmission is below a certain energy consumption threshold, and the remaining nodes are used to ensure that the smart nodes are in their design maintain normal operation during the life cycle, thereby reducing the proportion of total energy consumption.

Based on the comparison results of Figure 2 and Figure 3, it can be seen that the proposed method has obvious advantages in terms of energy saving and time delay reduction. In order to further verify effectiveness of this method, the optimal communication information wave domain test will be carried out. Figure 4 shows the optimal communication information wave domain test results under different methods.

It can be seen from Figure 4 that the optimal communication information wave domain of the communication node

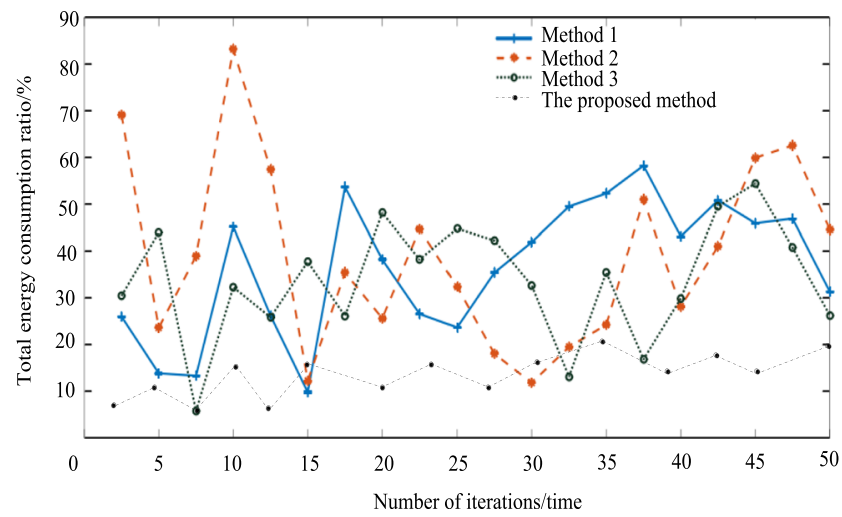

Fig 3. Comparison of the total energy consumption ratio of different methods 
selection method in this paper is stable, indicating that there is no serious interference node in selection process, and the optimal communication node can be selected stably under various IoT intrusions. The traditional optimal communication node selection method has serious wave pattern disorder in the wave domain, accompanied by certain wave level changes. The method in this paper considers the relation between the link maintenance time and the transmission delay, and establishes transmission characteristic model of the communication node according to the state evaluation result of the link between the nodes. This model is used to confirm the time sequence of frequency hopping signals and Internet of Things intrusion, and to mark the interfering nodes. Therefore, the optimal communication node selection method in this paper has strong anti-interference ability.

Through the above experiments, it can be concluded that the intelligent distance selection model proposed in this paper under the constraints of energy-saving IoT communication node selection can reduce the time delay when selecting IoT communication nodes, with better energy-saving performance and stable optimal communication information wave domain. The optimal communication node can be selected stably under various IoT intrusions. It can be seen that the optimal communication node selection method proposed in this paper has strong anti-interference ability and energy saving, and can be applied to the actual Internet of Things communication node selection process.

\section{Conclusion}

In order to solve problems of existing methods of delay ratio, high total energy consumption ratio, and disorder of optimal communication information waveband, a design method of intelligent spacing selection model under constraints of energy-saving communication node selection of the Internet of Things is proposed. According to energy-saving constraints, the link stability and link quality are evaluated, and interference node under intrusion of the Internet of Things is identified by determining transition amplitude of the noise node in transmission data sequence. On this basis, the distance between nodes is calculated, so as to realize the design of intelligent distance selection model for the communication nodes of the Internet of Things. The experimental results show that the method in this paper can quickly and accurately realize selection of the optimal node under the Internet of Things intrusion, has strong anti-interference ability, and comprehensive performance of this method is better than other current optimal communication node selection algorithms.

The method in this paper can realize intelligent selection of the distance between the communication nodes of the Internet

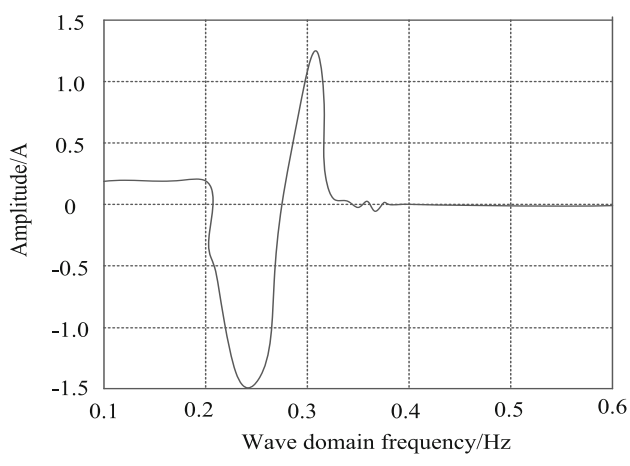

(a) Method 1

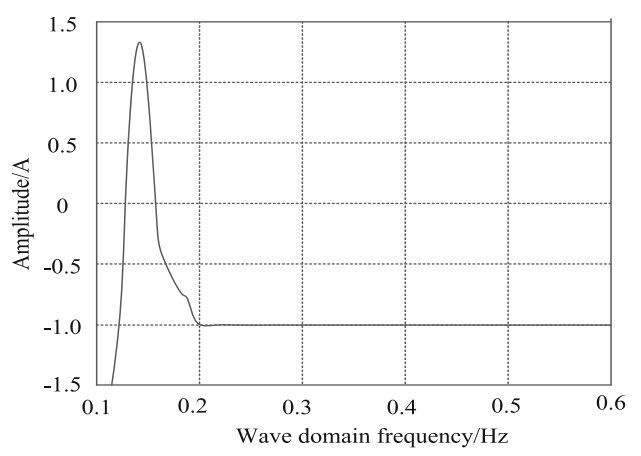

(b) Method 2

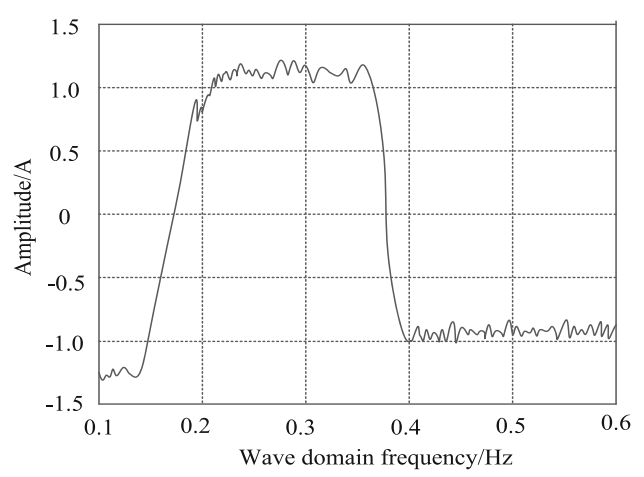

(c) Method 3

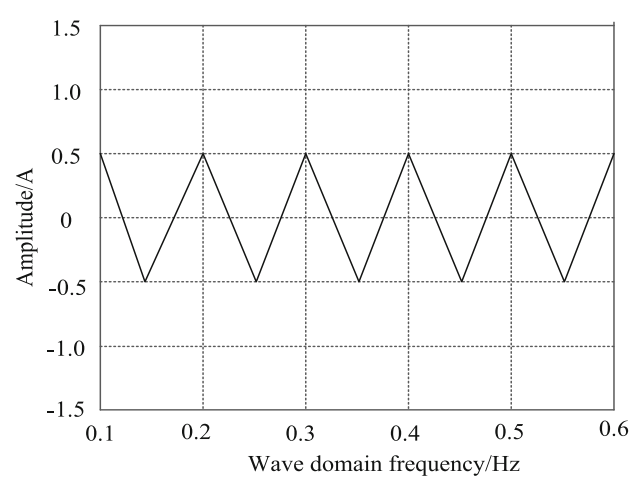

(d) The proposed method 
Fig 4. Wave domain test results of optimal communication information by different methods. (a) Method 1. (b) Method 2. (c) Method 3. (d) The proposed method.

of Things, but has not studied the energy consumption of some nodes, and lacks certain pertinence. Therefore, the energy consumption of a single node is taken as the research goal to improve the proposed method.

Open Access This article is licensed under a Creative Commons Attribution 4.0 International License, which permits use, sharing, adaptation, distribution and reproduction in any medium or format, as long as you give appropriate credit to the original author(s) and the source, provide a link to the Creative Commons licence, and indicate if changes were made. The images or other third party material in this article are included in the article's Creative Commons licence, unless indicated otherwise in a credit line to the material. If material is not included in the article's Creative Commons licence and your intended use is not permitted by statutory regulation or exceeds the permitted use, you will need to obtain permission directly from the copyright holder. To view a copy of this licence, visit http://creativecommons.org/licenses/by/4.0/.

\section{References}

1. Jeschke $\mathrm{S}$, Brecher $\mathrm{C}$, Song $\mathrm{H}$, and Rawat $\mathrm{D}$, Industrial Internet of Things. ISBN: 978-3-319-42558-0, Cham, Switzerland: Springer, 2017, pp. $1-715$

2. Ibrokhimov B, Hur C, Kang S (2020) Effective node selection technique towards sparse learning. Appl Intell 50(10):3239-3251

3. Wei W, Sun Z, Song H, Wang H, Fan X, Chen X (2018) Energy Balance-Based Steerable Arguments Coverage Method in WSNs. IEEE Access 6:33766-33773. https://doi.org/10.1109/ACCESS. 2017.2682845

4. Anand A, Conti M, Kaliyar P, Lal C (2020) TARE: Topology Adaptive Re-kEying scheme for secure group communication in IoT networks. Wirel Netw 26(4):2449-2463

5. Rawat AS, Koyluoglu OO, Vishwanath S (2018) Centralized Repair of Multiple Node Failures With Applications to Communication Efficient Secret Sharing. IEEE Trans Inf Theory 64(12):7529-7550

6. Biswash SK, Jayakody DNK (2020) Energy-efficient node-to-node communication scheme for fog-based cellular networks. IET Commun 14(10):1595-1602

7. Liu M (2017) Research on optimal Communication node selection method of optical fiber laser network after intrusion. Laser Journal 38(12): $154-158$

8. Wang L, Qian GB, Wang SY (2019) A distance aware and clustering assisted strategy for cellular-based IoT networks with energy harvesting. Journal of Chongqing University of Posts and Telecommunications (Natural Science Edition) 031(006):767-774

9. Shuai L, Shuai W, Xinyu L, et al. Human Memory Update Strategy: A Multi-Layer Template Update Mechanism for Remote Visual Monitoring, IEEE Transactions on Multimedia, 2021, online first, https://doi.org/10.1109/TMM.2021.3065580.

10. Ndashimye E, Sarkar NI, Ray SK (2020) A network selection method for handover in vehicle-to-infrastructure communications in multi-tier networks. Wirel Netw 26(1):387-401

11. Guido Dartmann, Houbing Song, and Anke Schmeink. Big Data Analytics for Cyber-Physical Systems: Machine Learning for the Internet of Things. ISBN: 9780128166376. Elsevier, 2019, pp. 1360 .

12. Bithas PS, Kanatas AG, Matolak DW (2019) Exploiting Shadowing Stationarity for Antenna Selection in V2V Communications. IEEE Trans Veh Technol 68(2):1607-1615

13. Jamshidi A (2019) Efficient cooperative ARQ protocols based on relay selection in underwater acoustic communication sensor networks. Wirel Netw 25(8):4815-4827

14. Khandaker MRA, Wong KK, Zheng G (2019) Truth-Telling Mechanism for Two-Way Relay Selection for Secrecy Communications With Energy-Harvesting Revenue. IEEE Trans Wirel Commun 16(5):3111-3123

15. Fucik R, Eichler P, Straka R, Paus P, Klinkovsky J, Oberhuber T (2019) On optimal node spacing for immersed boundary-lattice Boltzmann method in 2D and 3D. Comput Math Appl 77(4): 1144-1162

16. Shuai L, Chunli G, Fadi A et al (2020) Reliability of response region: A novel mechanism in visual tracking by edge computing for IIoT environments. Mech Syst Signal Process 138:106537

17. Liu S, Wang S, Liu X et al (2021) Fuzzy Detection aided Real-time and Robust Visual Tracking under Complex Environments. IEEE Trans Fuzzy Syst 29(1):90-102

18. Shridhar VS (2019) The India of Things: Tata Communications' countrywide IoT network aims to improve traffic, manufacturing, and health care. IEEE Spectr 56(2):42-47

19. Zhang Z (2019) Multi-Threaded Electronic Communication Network Peer-to-Peer Data Node Selection Simulation. Computer Simulation 036(011):276-279,326

20. Zewde TA, Gursoy MC (2019) Optimal Resource Allocation for Energy-Harvesting Communication Networks Under Statistical QoS Constraints. IEEE Journal on Selected Areas in Communications 37(2):313-326

21. Nanda A, Puthal D, Rodrigues JJPC, Kozlov SA (2019) Internet of Autonomous Vehicles Communications Security: Overview, Issues, and Directions. IEEE Wirel Commun 26(4):60-65

22. Sun Y, Song H, Jara AJ, Bie R (2016) Internet of Things and Big Data Analytics for Smart and Connected Communities. IEEE Access 4:766-773. https://doi.org/10.1109/ACCESS.2016. 2529723

Publisher's note Springer Nature remains neutral with regard to jurisdictional claims in published maps and institutional affiliations. 